\title{
DIGITALCOMMONS
}

@WAYNESTATE -

Wayne State University

$1-1-1984$

\section{Optimal Stochastic Scheduling of Power Generation Systems with Scheduling Delays and Large Cost Differentials}

\author{
G. L. Blankenship
}

University of Maryland, College Park

J.-L. Menaldi

Wayne State University, menaldi@wayne.edu

\section{Recommended Citation}

G. L. Blankenship and J.-L. Menaldi, Optimal stochastic scheduling of power generation systems with scheduling delays and large cost differentials, SIAM J. Control Optim., 22 (1984), pp. 121-132. doi: 10.1137/0322009

Available at: https://digitalcommons.wayne.edu/mathfrp/28 


\title{
OPTIMAL STOCHASTIC SCHEDULING OF POWER GENERATION SYSTEMS WITH SCHEDULING DELAYS AND LARGE COST DIFFERENTIALS*
}

\author{
G. L. BLANKENSHIP† AND J.-L. MENALDI $\ddagger$
}

\begin{abstract}
The optimal scheduling or unit commitment of power generation systems to meet a random demand involves the solution of a class of dynamic programming inequalities for the optimal cost and control law. We study the behavior of this optimality system in terms of two parameters: (i) a scheduling delay, e.g., the startup time of a generation unit; and (ii) the relative magnitudes of the costs (operating or starting) of different units. In the first case we show that under reasonable assumptions the optimality system has a solution for all values of the delay, and, as the delay approaches zero, that the solutions converge uniformly to those of the corresponding system with no delays. In the second case we show that as the cost of operating or starting a given machine increases relative to the costs of the other machines, there is a point beyond which the expensive machine is not used, except in extreme situations. We give a formula for the relative costs that characterize this point. Moreover, we show that as the relative cost of the expensive machine goes to infinity the optimal cost of the system including the expensive machine approaches the optimal cost of the system without the machine.
\end{abstract}

1. Introduction. Optimal scheduling of continuously evolving stochastic dynamical systems admitting costly, discrete state transitions as control actions involves the analysis of partial differential inequalities which constitute the dynamic programming optimality conditions for the problem. These are the "quasi-variational inequalities" (QVI's) introduced for such problems by A. Bensoussan and J. L. Lions [1] [2]. While there is an extensive analytical theory for the existence, uniqueness, and regularity properties of the solutions of QVI's, it is very difficult to describe the solutions and the associated optimal scheduling rules, i.e., the control laws, in any but the simplest cases. For this reason it is useful to examine the behavior of the solutions to QVI's as a function of various parameters which have simple interpretations in specific settings.

In this paper we consider the problem of scheduling a collection of power generation machines to meet a random demand for power, that is, the "unit commitment" problem. There are positive startup and operating costs associated with each machine, and the scheduling problem is to commit the units and operate them (set their power output levels) to meet the demand at minimum cost. The "demand" is modeled here as a diffusion process. In $\S 3$ we study the problem including scheduling delays in unit starting. (In power systems operations such delays correspond to the times for boiler reheating in steam turbine generators or crew travel times in manual start units [3].) In $\S 4$ we consider the scheduling problem when some machines are much more expensive to start and/or operate than any of the other machines.

Under reasonable assumptions on the demand dynamics and the cost functions we show that the optimality system (the QVI's) has a well-defined solution, cost and control policy, for all values of the scheduling delay, and, as the delay approaches zero, that the optimal cost converges uniformly to that of the corresponding system

\footnotetext{
* Received by the editors July 7, 1982, and in revised form August 15, 1982.

† Electrical Engineering Department, University of Maryland, College Park, Maryland 20742. The research of this author was supported in part by the U.S. Department of Energy under contract DE-AC0179ET29244.

$\ddagger$ Department of Mathematics, Wayne State University, Detroit, Michigan 48202. The research of this author was supported in part during a visit at the University of Maryland by the U.S. Department of Energy under contracts DE-AC01-79ET29244 and 01-80RA-50154; and in part by the Office of Sponsored Research Programs, Wayne State University.
} 
with no delay. The results of M. Robin [4] and J.-L. Menaldi [5] [6] form the basis for our arguments. In $\S 4$ we show that as the cost of starting and/or operating a designated machine increases relative to the costs of the other machines there is a point beyond which the expensive machine is not used, except in extreme situations. We give an inequality on the relative costs that characterizes this point. Moreover, we show that as the relative operating cost of the expensive machine goes to infinity the optimal cost of the system including the expensive machine approaches the optimal cost of the system excluding the machine.

Related work on the asymptotic analysis of QVI's in general and optimal scheduling problems in particular may be found in the papers [7]-[10] (among others). For the most part these are concerned with the asymptotic behavior as the noise intensity approaches zero, i.e., as the system dynamics reduce from stochastic to deterministic. The QVI's are, in such cases, singularly perturbed. The problems treated here are of a different type, although the case of large cost differentials has an order reduction effect in the asymptotic limit.

In [11] a result is given (Thm. 1.2, p. 192) which characterizes the optimal switching among alternatives in terms of a simple inequality on the costs. However, the problems considered in [11] do not include explicit costs for switching, and the methods used are quite different.

2. Problem statement and an existence result. Let $(\Omega, \mathscr{F}, P)$ be a probability space, $\left\{\mathscr{F}_{t}, t \geqq 0\right\}$ a nondecreasing, right-continuous family of completed sub- $\sigma$-fields of $\mathscr{F}$, and let $w(t), t \geqq 0$, be a standard $R^{N}$-valued Brownian motion with respect to $\mathscr{F}_{t}, t \geqq 0$.

Let $m \geqq 1$ be the number of machines. Let $A=\{0,1\}^{m}$ be the set of schedules. If $\mathbf{a} \in A$, and $a_{i}$ is the $i$ th element of $\mathbf{a}$, then $a_{i}=0$ means machine $i$ is down, and $a_{i}=1$ means it is up. Let $\left\{\theta_{j}, j=1,2, \cdots\right\}$ be an increasing sequence of stopping times with respect to $\mathscr{F}_{t}$ which are convergent to infinity and which satisfy $\theta_{j+1} \geqq \theta_{j}+h$, for each $j$ and some $h \geqq 0$, the scheduling delay. A scheduling policy a $(t), t \geqq 0$, is an $A$-valued random process starting at $\mathbf{a} \in \boldsymbol{A}$ and adapted to $\mathscr{F}_{t}$ satisfying

$$
\mathbf{a}(t)= \begin{cases}\mathbf{a}, & 0 \leqq t<\theta_{1}, \\ \mathbf{a}^{j}, & \theta_{j} \leqq t<\theta_{j+1}, \quad j=1,2, \cdots .\end{cases}
$$

Let $\mathscr{A}_{h, \mathrm{a}}$ be the set of all scheduling policies starting at a with delay $h$. These are the discrete controls for our system. The components $a_{i}(t), i=1,2, \cdots, m$, of $\mathbf{a}(t)$ are the unit commitment schedules for the individual machines. Let $\left[\check{p}_{i}, \hat{p}_{i}\right] \subset(0, \infty)$, $i=1, \cdots, m$, be the output capacities of the machines when up, and let $P=\left[\check{p}_{1}, \hat{p}_{1}\right] \times \cdots \times\left[\check{p}_{m}, \hat{p}_{m}\right] \subset R^{m}$. Then $\mathbf{p} \in P$ is the vector of power generations from the ensemble of machines. The system control-the power production-is

$$
\boldsymbol{v}(t)=\mathbf{a}(t) \circ \mathbf{p}(t)=\left\{a_{j}(t) p_{j}(t), j=1, \cdots, m\right\}
$$

for $\mathbf{a}(t) \in \mathscr{A}_{h, \mathbf{a}}, p(\cdot):[0, \infty] \rightarrow P$. We have used the Schur product notation in (2.2). Let $P_{0}=\left[0, \check{p}_{1}\right] \times \cdots \times\left[0, \check{p}_{m}\right]$ be the output powers of the ensemble of machines including the possibility of shutdowns.

Now let $g(x, \mathbf{a}), \sigma(x, \mathrm{a})$ be two given functions on $R^{N} \times A$ into $R^{N}$ and $R^{N} \otimes R^{N}$, respectively, which are Lipschitz continuous in $x$ for each fixed a, $g=\left(g_{i}\right), \sigma=\left(\sigma_{i j}\right)$,

$$
\frac{\partial g_{i}}{\partial x_{k}}, \frac{\partial \sigma_{i j}}{\partial x_{k}} \in B\left(R^{N}\right), \quad i, j, k=1, \cdots, N \quad \forall \mathbf{a} \in A
$$

where $B\left(R^{N}\right)$ is the set of $R$-valued, Borel measurable, bounded functions on $R^{N}$. 
The $R^{N}$-valued diffusion $y(t)=y_{x}(t, \mathbf{a}(\cdot))$ with drift $g$ and diffusion $\sigma \sigma^{T}$ characterizes the demand on the system. We permit the demand to depend on the schedule. Let $\{\mathbf{a}(t), t \geqq 0\} \in \mathscr{A}_{h, \mathbf{a}}$ and

$$
d y(t)=g[y(t), \mathbf{a}(t)] d t+\sigma[y(t), \mathbf{a}(t)] d w(t), \quad y(0)=x \in R^{N}, \quad t \geqq 0 .
$$

The process $y$ has continuous paths almost surely.

Let $\mathcal{O}$ be a bounded subset of $R^{N}$ and let $\overline{\mathcal{O}}$ be its closure. We denote by $\tau=\tau_{x}(\mathbf{a}(\cdot))$ the first exit time of $y_{x}(t, \mathbf{a}(\cdot))$ from $O$. That is,

$$
\tau_{x}(a(\cdot))=\inf \left\{t \geqq 0: y_{x}(t, \mathbf{a}(\cdot)) \notin \overline{\mathcal{O}}\right\}
$$

for each $\mathbf{a} \in A$ and $\mathbf{a}(\cdot) \in \mathscr{A}_{h, \mathbf{a}}$. (Recall $\mathbf{a} \in A$ is $\mathbf{a}(0)$ for $\left.\mathbf{a}(\cdot) \in \mathscr{A}_{h, \mathbf{a}}\right)$ Let $\Gamma_{\mathbf{a}}$ be the set of regular points of $\partial \mathcal{O}$ from a (cf. [12])

$$
\Gamma_{\mathrm{a}}=\left\{x \in \partial \mathcal{O}: P\left(\tau_{x, \mathbf{a}}>0\right)=0\right\}
$$

where $\mathbf{a}$ is a constant scheduling policy. If $\left\{\theta_{i}, i=1,2, \cdots\right\}$ is a sequence of stopping times, $\mathbf{a} \in A \mathbf{a}(\cdot) \in \mathscr{A}_{h, \mathbf{a}}$, and $\{\mathbf{p}(t), t \geqq 0\}$ is a $P$-valued process adapted to $\mathscr{F}_{t}$ with right continuous trajectories (having left-hand limits), then $\{\boldsymbol{v}(t)=\mathbf{a}(t) \circ \mathbf{p}(t), t \geqq 0\}$ is called an admissible control. We have

$$
y(\tau, \mathbf{a}) \in \Gamma_{\mathbf{a}} \quad \text { a.s. on }\{\tau<\infty\} \quad \forall \mathbf{a} \in A .
$$

The cost functional for the problem is defined as follows: let $f: R^{N} \times P_{0} \rightarrow(0, \infty)$ be continuous; $f$ is the operating cost rate. The switching cost $k: A \times A \rightarrow[0, \infty)$ is

$$
k(\mathbf{a}, \mathbf{b})=\sum_{j=1}^{m} k_{j}\left[b_{j}-a_{j}\right]^{+}, \quad \mathbf{a}, \mathbf{b} \in \boldsymbol{A}
$$

where $k_{j} \geqq k_{0}>0$ for $j=1, \cdots, m$. The cost is

$$
J_{x \mathbf{a}}(\boldsymbol{v})=E_{x \mathbf{a}}\left\{\int_{0}^{\tau} f[y(t, \mathbf{a}(\cdot)), \mathbf{v}(t)] e^{-\alpha t} d t+\sum_{i=1}^{\infty} k\left[\mathbf{a}\left(\theta_{i-1}\right), \mathbf{a}\left(\theta_{i}\right)\right] \mathbf{1}_{\theta_{i}<\tau} e^{-\alpha \theta_{i}}\right\}
$$

where $\alpha>0$ is the discount factor, $E_{x \mathbf{a}}\{\cdot\}$ is expectation over paths $y(t), \mathbf{a}(t)$ starting in $x \in R^{N}$ and $\mathbf{a} \in A$, respectively, and $\theta_{0}=0$.

Problem statement. We wish to characterize the optimal cost

$$
u_{h}(x, \mathbf{a})=\inf \left\{J_{x \mathbf{a}}(\boldsymbol{v}): \boldsymbol{v} \text { admissible }\right\}
$$

as a function of the scheduling delay $h$ and the relative costs $f(y, \mathbf{p} \circ \mathbf{a}) / f(y, \mathbf{p} \circ \mathbf{b})$, $k(\mathbf{a}, \mathbf{b}) / k(\mathbf{a}, \mathbf{c})$ for all $\mathbf{a}, \mathbf{b}, \mathbf{c} \in A$.

The first question of interest is the existence of the optimal cost. Since the problem is possibly degenerate $\left(\operatorname{det} \sigma \sigma^{T}(x, \mathbf{a})=0\right.$ for some $\left.x, \mathbf{a}\right)$ and irregular $\left(\Gamma_{\mathbf{a}}\right.$ not closed), this is a potentially delicate issue. However, the results of [5] and [6] adapt to the present case with minor modifications. Since we are mainly interested in the qualitative features of the optimal scheduling problem, we shall present a minimal treatment of the existence question.

For each $\mathbf{a} \in A$ we associate the operators

$$
\mathscr{L}_{\mathrm{a}}=-\frac{1}{2} \operatorname{tr}\left[\sigma \sigma^{\mathrm{T}} \partial_{\mathrm{x} x}\right]-g \cdot \partial_{x}, \quad \tilde{\mathscr{L}}_{\mathbf{a}}=\mathscr{L}_{\mathrm{a}}+\alpha,
$$

with the diffusion

$$
d y^{0}(t)=g\left(y^{0}(t), \mathbf{a}\right) d t+\sigma\left(y^{0}(t), \mathbf{a}\right) d w(t)
$$


(Here a plays the role of a parameter.) Following [5] and [6] we use the integral formulation of $\hat{\mathscr{L}}_{\mathbf{a}}$; that is,

$$
\hat{\mathscr{L}}_{\mathbf{a}} u(x, \mathbf{a}) \leqq \hat{f}(x, \mathbf{a}) \quad \text { in } \overline{\mathcal{O}}-\Gamma_{\mathbf{a}}
$$

if the process

$$
x_{t}=\int_{0}^{\theta \wedge \tau^{0}} \hat{f}\left(y_{x}^{0}(s), \mathbf{a}\right) e^{-\alpha s} d s+u\left(y_{x}^{0}\left(t \wedge \tau^{0}\right), \mathbf{a}\right) e^{-\alpha\left(t \wedge \tau^{0}\right)}
$$

is a $\mathscr{F}_{t}$-submartingale for each $x \in \overline{\mathcal{O}}-\Gamma_{\mathrm{a}}$.

Here

$$
\hat{f}(x, \mathbf{a})=\min \{f(x, \mathbf{p} \circ \mathbf{a}), \mathbf{p} \in P\} .
$$

We shall also say that $\hat{\mathscr{L}}_{\mathrm{a}} u \leqq \hat{f}$ in the martingale sense when (2.13) holds.

Define the operator $M$ as

$$
\begin{aligned}
M u(x, \mathbf{a})=\min _{\mathbf{b} \neq \mathbf{a}} E_{x \mathbf{b}}\left\{\int_{0}^{h \wedge \delta} f(y(t, \mathbf{b}), \mathbf{b}) e^{-\alpha t} d t\right. \\
\left.+k(\mathbf{a}, \mathbf{b})+e^{-\alpha(h \wedge \tau)} u(y(h \wedge \tau, \mathbf{b}), \mathbf{b})\right\} .
\end{aligned}
$$

If we set $\|v\|=\sup \{|v(x, \mathbf{a})|, x \in \overline{\mathcal{O}}, \mathbf{a} \in A\}$ for $v(\cdot, \mathbf{a})$ continuous on $\overline{\mathcal{O}}$, then $M$ maps $C(\bar{O})$ into itself and

$$
\|M u-M v\| \leqq e^{-\alpha h}\|u-v\|,
$$

if $u(x, \mathbf{a})=0=v(x, \mathbf{a})$ for all $\mathbf{x} \in \Gamma_{\mathbf{a}}, \mathbf{a} \in A$.

The problem (2.10) can be formulated as follows.

Find a real, bounded, measurable function $u(x, \mathbf{a})$ on $\bar{O} \times A$ such that

$$
\begin{aligned}
& u=0 \quad \text { on } \Gamma_{\mathbf{a}}, \quad \forall \mathbf{a} \in A, \\
& u \leqq M u \quad \text { in } \overline{\mathcal{O}}-\Gamma_{\mathbf{a}}, \quad \forall \mathbf{a} \in A, \\
& \hat{\mathscr{L}}_{\mathbf{a}} u \leqq \hat{f} \text { in the martingale sense on } \overline{\mathcal{O}}-\Gamma_{\mathbf{a}}, \quad \forall \mathbf{a} \in A .
\end{aligned}
$$

We can reformulate the problem (2.10) or (2.17) as a quasi-variational inequality along the lines in [6, p. 724], but this takes us somewhat away from our main line of inquiry, and so, we will omit it.

We associate with (2.10) a sequence of stopping time problems as follows. Let

$$
\hat{u}^{0}(x, \mathbf{a})=E_{x \mathbf{a}}\left\{\int_{0}^{\tau} \hat{f}(y(t, \mathbf{a}), \mathbf{a}) e^{-\alpha t} d t\right\} .
$$

Given $\hat{u}^{n-1}(x, \mathbf{a})$, define $\hat{u}^{n}(x, \mathbf{a})$ by

$$
\hat{u}^{n}(x, \mathbf{a})=\inf _{\theta \geqq 0} E_{x \mathbf{a}}\left\{\int_{0}^{\theta \wedge \tau} \hat{f}\left(y_{x}(t, \mathbf{a}), \mathbf{a}\right) e^{-\alpha t} d t+\mathbf{1}_{\theta<\tau} e^{-\alpha \theta} M \hat{u}^{n-1}\left(y_{x}(\theta, \mathbf{a}), \mathbf{a}\right)\right\}
$$

Note that $\mathbf{a} \in A$ is a parameter in (2.18), (2.19). In abstract terms (2.18), (2.19) takes the following equivalent form: let $\hat{u}^{0}(x, a)$ be the bounded, continuous, nonnegative real function on $\bar{O} \times A$ such that

$$
\begin{aligned}
& \hat{u}^{0}(x, \mathbf{a})=0 \quad \forall x \in \Gamma_{\mathbf{a}}, \quad \forall \mathbf{a} \in A, \\
& \hat{\mathscr{L}}_{\mathbf{a}} \hat{u}^{0}=\hat{f} \text { in the martingale sense on } \overline{\mathcal{O}}-\Gamma_{\mathbf{a}} \quad \forall \mathbf{a} \in A,
\end{aligned}
$$


and, given $\hat{u}^{n-1}$, let $\hat{u}^{n}$ be the bounded, continuous, nonnegative real function on $\overline{\mathcal{O}}$ which is the maximum solution of

$$
\begin{aligned}
& u^{n}(x, \mathbf{a})=0 \quad \forall x \in \Gamma_{\mathbf{a}} \quad \forall \mathbf{a} \in A, \\
& u^{n} \leqq M \hat{u}^{n-1} \quad \text { in } \overline{\mathcal{O}} \Gamma_{\mathbf{a}}, \\
& \hat{\mathscr{L}}_{\mathbf{a}} u^{n} \leqq \hat{f} \text { in the martingale sense on } \overline{\mathcal{O}}-\Gamma_{\mathbf{a}} \quad \forall \mathbf{a} \in A .
\end{aligned}
$$

The sequence of variational inequalities corresponds, in effect, to the sequence of stopping time problems-make $n$ optimal decisions, startup or shutdown, and then stop.

LEMMA 2.1. Under the stated hypotheses on $g, \sigma, f$, and $k$ the problem (2.17) admits a maximum solution $\hat{u}$ which is upper semicontinuous and given as the optimal cost in (2.10). Moreover,

$$
\begin{gathered}
0 \leqq \hat{u}^{n+1} \leqq \hat{u}^{n} \leqq \cdots \leqq \hat{u}^{0} \leqq \frac{1}{\alpha}\|\hat{f}\| \quad \forall n=1,2, \cdots, \\
0 \leqq \hat{u}=\hat{u}^{n} \leqq\left[\frac{\exp (-n \alpha h)}{1-\exp (-\alpha h)}\right]\left\|\hat{u}^{1}-\hat{u}^{0}\right\|,
\end{gathered}
$$

and if the set of regular points $\Gamma_{a}$ is closed, then

$$
\hat{u}^{n}(\cdot, \mathbf{a}), \hat{u}(\cdot, \mathbf{a}) \in C .
$$

Proof. The first two results (2.22) and (2.23) follow from simple modifications of the arguments in Robin [4, pp. 279-283]. The third result (2.24) follows from the arguments in [5]. QED

THEOREM 2.1. Under the stated hypotheses on $f, g, \sigma$, and $k$ and the assumption of regularity $\left(\Gamma_{a}\right.$ closed $\left.\forall \mathbf{a}\right)$ there exists an optimal, admissible control policy.

Proof. First, note that $\hat{u}(x, \mathbf{a})$ constructed as the limit of the sequence (2.19) via (2.23) satisfies the problem

$$
\hat{u}(x, \mathbf{a})=\inf _{l \geqq 0} E_{x, \mathbf{a}}\left\{\int_{0}^{\theta \wedge \tau} \hat{f}(y(t, \mathbf{a}), \mathbf{a}) e^{-\alpha t} d t+\mathbf{1}_{\theta<\tau} e^{-\alpha \theta} \boldsymbol{M} \hat{u}(y(\theta, \mathbf{a}), \mathbf{a})\right\} .
$$

Let $\hat{b}(x, \mathbf{a})$ be defined by

$$
\begin{aligned}
\hat{b}(x, \mathbf{a})=\underset{\mathbf{b} \neq \mathbf{a}}{\arg \min }\left[k(\mathbf{a}, \mathbf{b})+E_{x \mathbf{b}}\left\{\int_{0}^{h \wedge \tau} \hat{f}(y(t, \mathbf{b}), \mathbf{b}) e^{-\alpha} t d t\right.\right. \\
\left.\left.+e^{-\alpha(h \wedge \tau)} u(y(h \wedge \tau, \mathbf{b}), \mathbf{b})\right\}\right]
\end{aligned}
$$

with $\hat{b}$ Borel measurable in $\overline{\mathcal{O}} \times A$.

The optimal policy $\hat{\mathbf{a}}(\cdot)$ is defined by

$$
\hat{\mathbf{a}}(\cdot)=\mathbf{a} \mathbf{1}_{\left[0, \hat{\theta}_{1}\right)}+\sum_{i=1}^{\infty} \hat{\mathbf{a}}^{i} \mathbf{1}_{\left[\hat{\theta}_{i}, \hat{\theta}_{i+1}\right)}
$$

with the values $\hat{\mathbf{a}}^{i}$ selected as follows. Let

$$
\tilde{\boldsymbol{\theta}}^{0}=0
$$

$$
d \hat{y}^{0}(t)=g\left(\hat{y}^{0}(t), \mathbf{a}\right) d t+\sigma\left(\hat{y}^{0}(t), \mathbf{a}\right) d w(t), \quad \hat{y}^{0}(0)=x, \quad t \geqq 0,
$$


and for $i=0,1,2, \cdots$

$$
\hat{\tau}^{i}=\left\{\begin{array}{l}
\inf \left\{t \geqq 0: \hat{y}^{i}(t) \in \mathcal{O}\right\} \\
\infty \text { if the set is empty }
\end{array}\right.
$$$$
\hat{\theta}_{i+1}=\left\{\begin{array}{l}
\inf \left\{t \in\left[\tilde{\theta}^{i}, \hat{\tau}^{i}\right): \hat{y}^{i}(t) \notin\left\{\hat{u}\left(\circ, \hat{\mathbf{a}}^{i}\right)<M \hat{u}\left(\circ, \hat{\mathbf{a}}^{i}\right)\right\}\right\} \\
\infty \text { if the set is empty, }
\end{array}\right.
$$

$$
\begin{aligned}
& \tilde{\theta}^{i}=\left(\hat{\theta}_{i}+h\right) \wedge \hat{\tau}^{i}, \quad i=1,2, \cdots, \\
& \hat{\mathbf{a}}^{i+1}= \begin{cases}\hat{b}\left(\hat{y}^{i}\left(\hat{\theta}_{i+1}\right), \hat{\mathbf{a}}^{i}\right), & \text { if } \hat{\theta}_{i+1}<\infty \\
\hat{\mathbf{a}}^{i}, & \text { otherwise, } \quad i=0,1,2, \cdots,\end{cases}
\end{aligned}
$$

and

$$
\begin{aligned}
& d \hat{y}^{i}(t)=g\left(\hat{y}^{i}(t), \hat{\mathbf{a}}^{i}\right) d t+\sigma\left(\hat{y}^{i}(t), \hat{\mathbf{a}}^{i}\right) d w(t), \quad t \geqq \hat{\theta}_{i}, \\
& \hat{y}^{i}(t)=\hat{y}^{i-1}(t), \quad t \leqq \hat{\theta}_{i}, \quad i=1,2, \cdots .
\end{aligned}
$$

Using the Markov property, we have

$$
\begin{aligned}
u(x, \mathbf{a})= & E\left\{\int_{0}^{\hat{\theta}_{n} \wedge \hat{\tau}^{n-1}} \hat{f}(\hat{y}(t), \hat{\mathbf{a}}(t)) e^{-\alpha t} d t+\sum_{i=1}^{n} k\left(\hat{\mathbf{a}}^{i-1}, \hat{\mathbf{a}}^{i}\right) e^{-\alpha \hat{\theta}_{i}} \mathbf{1}_{\hat{\theta}_{i}<\hat{r}}\right\} \\
& +E\left\{e^{-\alpha \hat{\theta}_{n}} \hat{u}\left(\hat{y}\left(\hat{\theta}_{n}\right), \hat{\mathbf{a}}^{n}\right)\right\},
\end{aligned}
$$

where

$$
\hat{y}(t)=y(t, \hat{\mathbf{a}}(\cdot))=\hat{y}^{n}(t) \quad \forall t \in\left[0, \hat{\theta}_{n}\right] .
$$

Since $\hat{u}$ is bounded and $\hat{\theta} \rightarrow \infty$ (a.s.) as $n \rightarrow \infty$, we obtain

$$
u(x, \mathbf{a})=E\left\{\int_{0}^{\hat{\tau}} \hat{f}(\hat{y}(t), \hat{\mathbf{a}}(t)) e^{-\alpha t} d t+\sum_{i=1}^{\infty} k\left(\hat{\mathbf{a}}^{i-1}, \hat{\mathbf{a}}^{i}\right) e^{-\alpha \hat{\theta}_{i}} \mathbf{1}_{\hat{\theta}_{i}<\hat{\tau}}\right\} .
$$

Finally, let $\hat{\mathbf{p}}(x, \mathbf{a})$ measurable be such that

$$
f(x, \mathbf{a} \circ \hat{\mathbf{p}}(x, \mathbf{a}))=\hat{f}(x, \mathbf{a}) \quad \forall(x, \mathbf{a}) \in \overline{\mathcal{O}} \times A .
$$

Defining

$$
\hat{\mathbf{p}}(t)=\hat{\mathbf{p}}(y(t), \hat{a}), \quad \hat{\boldsymbol{v}}(t)=\hat{\mathbf{p}}(t) \circ \hat{\mathbf{a}}(t)
$$

completes the proof. QED

Remark. The function $\hat{u}^{n}(x, \mathbf{a})$ is the optimal cost given that $n$ switchings are permitted.

3. Dependence of the cost on the scheduling delay. In this section we shall show that the optimal scheduling cost depends continuously on the delay $h$ as $h \rightarrow 0$, if the hypotheses of Theorem 2.1 hold. To emphasize the dependence on $h$, let $\hat{u}_{h}(x, a)$ be the optimal cost in the problem (2.10), and let $\hat{u}_{h}^{n}$ be the costs in the sequence (2.19). Also, let

$$
M_{h} v(x, \mathbf{a})=\min _{\mathbf{b} \neq \mathbf{a}}\left[k(\mathbf{a}, \mathbf{b})+E_{x \mathbf{b}}\left\{\int_{0}^{h \wedge \tau} \hat{f}(y(t, \mathbf{b}), \mathbf{b}) e^{-\alpha t} d t+e^{-\alpha(h \wedge \tau)} v(y(t, \mathbf{b}), \mathbf{b})\right\}\right]
$$

and

$$
\operatorname{Mv}(x, \mathbf{a})=\min _{\mathbf{b} \neq \mathbf{a}}[k(\mathbf{a}, \mathbf{b})+v(x, \mathbf{a})]
$$


LEMMA 3.1. Under the stated hypotheses on $f, g, \sigma$ and $k$ and the assumption of regularity we have

$$
\left\|M_{h} v-M v\right\| \leqq\left(\frac{1-e^{-\alpha h}}{\alpha}\right)\|\hat{f}\|+\left(1-e^{-\alpha h}\right)\|v\|
$$

for all Borel measurable $v$ such that

$$
v(x, \mathbf{a})=0 \quad \forall x \in \Gamma_{\mathbf{a}}, \quad \forall \mathbf{a} \in A .
$$

Proof. This follows immediately from (3.1) (3.2). QED LEMMA 3.2. Under the conditions of Lemma 3.1 we have

$$
\left\|\hat{u}_{h}^{n}-\hat{u}_{h}\right\| \leqq \frac{c}{n-m}, \quad n=m+1, \quad m+2, \cdots
$$

for some constant $c$ independent of $h$ and where $m$ is the number of machines.

Proof. Let

$$
\begin{aligned}
& N^{+}[0, T]=\text { number of machine starts in }[0, T], \\
& N^{-}[0, T]=\text { number of machine shutdowns in }[0, T]
\end{aligned}
$$

associated with a policy $\mathbf{a}(t), t \in[0, T]$. For each $T>0$

$$
N^{+}[0, T]+N^{-}[0, T] \leqq 2 N^{+}[0, T]+m .
$$

Given any policy $\mathbf{a}(\cdot)$, let $\mathbf{a}^{n}(\cdot)$ be the policy whose first $n$ switchings coincide with those of $\mathbf{a}(\cdot)$ and which is constant (at the value of the $n$th switching) throughout the remainder of the interval. Using the notation $\hat{u}_{h}^{n}$ introduced earlier, we have

$$
0 \leqq \hat{u}_{h}^{n}-\hat{u}_{h} \leqq \sup _{\mathbf{a}(\cdot)} E\left\{\int_{\theta_{n} \wedge \tau}^{\tau} \hat{f}\left(y^{n}(t), \mathbf{a}^{n}(t)\right) e^{-\alpha t} d t\right\},
$$

and so,

$$
0 \leqq \hat{u}_{h}^{n}-\hat{u}_{h} \leqq\|\hat{f}\| \sup _{\mathbf{a}(\cdot)} E\left\{e^{-\alpha\left(\theta_{n}^{\wedge}\right)} \mathbf{1}_{\theta_{n}<\tau}\right\} .
$$

To estimate the expectation, we use (3.7) and an observation about the startup cost. Clearly, for any $T>0$

$$
k_{0} N^{+}[0, T] e^{-\alpha T} \leqq \sum_{0 \leqq t \leqq T} k\left[\mathbf{a}\left(t_{-}\right), \mathbf{a}(t)\right] e^{-\alpha} t .
$$

Now from (3.7) for a policy with $n$ switching

$$
n \leqq 2 N^{+}\left[0, \theta_{n}\right]+m \text {. }
$$

Using this in (3.10) with $T=\theta_{n} \wedge \tau$, we have

$$
\frac{1}{2} k_{0}(n-m) E\left\{e^{-\alpha\left(\theta_{n} \wedge \tau\right)} \mathbf{1}_{\theta_{n}<\tau}\right\} \leqq \sup _{\mathbf{a}(\cdot)} E\left\{\sum_{i=1}^{\infty} k\left[\mathbf{a}\left(\theta_{i-1}\right), \mathbf{a}\left(\theta_{i}\right)\right] e^{-\alpha\left(\theta_{i} \wedge \tau\right)}\right\} .
$$

To estimate the term on the right, recall from (2.9), the form of the cost of an admissible policy. Consider a suboptimal policy which involves no switching. The cost of such a policy is bounded above by $\|\hat{f}\| / \alpha$. It follows from (2.9) that we can restrict attention to policies in which

$$
E\left\{\sum_{0 \leqq t \leqq \tau} k[\mathbf{a}(t-), \mathbf{a}(t)] e^{-\alpha t}\right\} \leqq\|\hat{f}\| / \alpha .
$$


Using this on the right in (3.12), and the result in (3.9), we have the desired inequality (3.5) with

$$
c=2\|\hat{f}\|^{2} / \alpha k_{0}
$$

The bound (3.5) and an inequality of Robin give us the desired continuity result. Let $\hat{u}_{0}(x$, a) be the optimal cost in the system (2.10) with no delay, i.e., $h=0$.

THEOREM 3.1. Under the stated hypotheses on $f, g, \sigma$, and $k$ and the assumption of regularity we have

$$
\lim _{h \downarrow 0} \hat{u}_{h}=\hat{u}_{0} \quad \text { uniformly in } \overline{\mathcal{O}} \times A .
$$

Proof. Let $\hat{u}_{0}^{n}$ be the optimal cost in the problem (2.10) with no delay over admissible policies having at most $n$ switchings. Then

$$
\left\|\hat{u}_{h}-\hat{u}_{0}\right\| \leqq\left\|\hat{u}_{h}-\hat{u}_{h}^{n}\right\|+\left\|\hat{u}_{h}^{n}-\hat{u}_{0}^{n}\right\|+\left\|\hat{u}_{0}^{n}-\hat{u}_{0}\right\| .
$$

The first and third terms on the right may be bounded using Lemma 3.1. A bound for the second term is given in Robin's thesis [4], p. 235,

$$
\left\|\hat{u}_{h}^{n}-\hat{u}^{n}\right\| \leqq 2 n h\|\hat{f}\|
$$

It follows that for any $n \geqq m+1$

$$
\left\|\hat{u}_{h}-\hat{u}_{0}\right\| \leqq\left(4\|\hat{f}\|^{2} / \alpha k_{0}\right) /(n-m)+2\|\hat{f}\| n h .
$$

Thus, taking $h \downarrow 0$ and then $n \rightarrow \infty$ leads to the desired result. QED

4. Scheduling with some expensive machines. Now suppose that one machine, or more generally, a group of machines is much more expensive to operate and/or start than the remaining machines. One would expect that the expensive machine would be used only in extreme circumstances, or not at all when its cost is very high. We show that the problem $(2.10)$ has these properties.

Let $\mathbf{a}^{e} \in A$ be an "expensive" schedule. Recall the notation $\hat{f}(x, \mathbf{a})=$ $\min \{f(x, \mathbf{p} \circ a), \mathbf{p} \in P\}$.

LEMMA 4.1. Under the stated hypotheses on $f, g, \sigma$ and $k$ and the assumption of regularity the inequality

$$
\hat{f}\left(x, \mathbf{a}^{e}\right)>\hat{f}(x, \mathbf{b})+\left[k(\mathbf{a}, \mathbf{b})-k\left(\mathbf{a}, \mathbf{a}^{e}\right)\right]\left(\frac{\alpha}{1-e^{-\alpha h}}\right) \quad \forall x \in \overline{\mathcal{O}}, \mathbf{a} \in A, \mathbf{b} \in A-\left\{\mathbf{a}^{e}\right\}
$$

implies that the optimal policy $\hat{a}(t), 0 \leqq t \leqq \hat{\tau}$, defined in Theorem 2.1 for the problem (2.10) has the property

$$
P\left\{\hat{\mathbf{a}}(t)=\mathbf{a}^{e}, 0 \leqq t \leqq \hat{\tau}-h\right\}=0 .
$$

Remark. In other words, the optimal policy $\hat{\mathbf{a}}(\cdot)$ switches to the expensive schedule only near the boundary $\Gamma$, and in that case, it switches once and then stops.

Proof. Let $\mathbf{a}(\cdot)$ be the optimal policy. Over an interval $\left[\hat{\theta}_{i}, \hat{\theta}_{i+1}\right)$ the optimal cost increases by the amount

$$
\Delta J_{i}=\int_{\hat{\theta}_{i}}^{\hat{\theta}_{i+1}} \hat{f}\left(\hat{y}(t), \hat{\mathbf{a}}^{i}\right) e^{-\alpha t} d t+k\left(\hat{\mathbf{a}}^{i-1}, \hat{\mathbf{a}}^{i}\right) e^{-\alpha \hat{\theta}_{i}}
$$


Suppose that $\hat{\mathbf{a}}^{i-1} \neq \mathbf{a}^{e}$ and $\hat{\mathbf{a}}^{i}=\mathbf{a}^{e}$. Then by (4.1) for any $\mathbf{b} \in A-\left\{\mathbf{a}^{e}\right\}$ we have

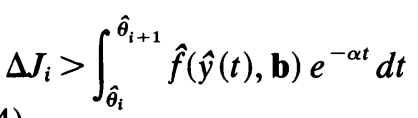

$$
+\left(\frac{1}{1-e^{-\alpha h}}\right)\left(e^{-\alpha \hat{\theta}_{i}}-e^{-\alpha \hat{\theta}_{i+1}}\right)\left[k\left(\hat{\mathbf{a}}^{i-1}, \mathbf{b}\right)-k\left(\hat{\mathbf{a}}^{i-1}, \mathbf{a}^{e}\right)\right]+k\left(\hat{\mathbf{a}}^{i-1}, \mathbf{a}^{e}\right) e^{-\alpha \hat{\theta}_{i}} .
$$

Since $\hat{\theta}_{i+1} \geqq h+\hat{\theta}_{i}$ if $0 \leqq t \leqq \hat{\tau}-h$, it follows that

$$
\Delta J_{i}>\int_{\hat{\theta}_{i}}^{\hat{\theta}_{i+1}} \hat{f}(\hat{y}(t), \mathbf{b}) e^{-\alpha t} d t+k\left(\hat{\mathbf{a}}^{i-1}, \mathbf{b}\right) e^{-\alpha \hat{\theta}_{i}},
$$

and this must have probability zero since $\hat{\mathbf{a}}(t)$ is optimal. QED

Note that either a large "operating cost rate" $\hat{f}\left(x, \mathbf{a}^{e}\right)$ or a large "startup cost" $k\left(\mathbf{a}, \mathbf{a}^{e}\right)$ will cause (4.1) to be satisfied. Now suppose that the operating cost $\hat{f}\left(x, \mathbf{a}^{e}\right)$ becomes arbitrarily large

$$
\hat{f}\left(x, \mathbf{a}^{e}\right) \geqq 1 / \varepsilon, \quad x \in \overline{\mathcal{O}}, \quad \varepsilon>0 \text { small. }
$$

One would expect that in the limit as $\varepsilon \downarrow 0$ that the expensive state $\mathbf{a}^{e}$ will never be used. We shall treat the cases $h>0$ and $h=0$ separately. Let

$$
\begin{aligned}
& \hat{u}_{h}^{e}(x, \mathbf{a})=\inf \left\{J_{x \mathbf{a}}(\boldsymbol{v}): \boldsymbol{v}(t)=\mathbf{p}(t) \circ \mathbf{a}(t), \mathbf{a}(t) \neq \mathbf{a}^{e}, 0 \leqq t \leqq \hat{\tau}, h>0\right\}, \\
& \hat{u}_{0}^{e}(x, \mathbf{a})=\inf \left\{J_{x \mathbf{a}}(\boldsymbol{v}): \boldsymbol{v}(t)=\mathbf{p}(t) \circ \mathbf{a}(t), \mathbf{a}(t) \neq \mathbf{a}^{e}, 0 \leqq t \leqq \hat{\tau}, h=0\right\} .
\end{aligned}
$$

Let

$$
\begin{aligned}
& \hat{u}_{h}^{\varepsilon}(x, \mathbf{a})=\inf \left\{J_{x \mathbf{a}}(\boldsymbol{v}): \boldsymbol{v}(\cdot) \text { admissible, (4.6) holds, } h>0\right\}, \\
& \hat{u}_{0}^{\varepsilon}(x, \mathbf{a})=\inf \left\{J_{x \mathbf{a}}(\boldsymbol{v}): \boldsymbol{v}(\cdot) \text { admissible, (4.6) holds, } h=0\right\} .
\end{aligned}
$$

THEOREM 4.1. Under the stated hypotheses on $f, g, \sigma$ and $k$, the assumption of regularity then

$$
\lim _{\varepsilon \downarrow 0} \hat{u}_{h}^{\varepsilon}=\hat{u}_{h}^{e} \quad \text { uniformly in } x \in \overline{\mathcal{O}}, \quad h>0 .
$$

If, in addition,

$$
g=g(x), \quad \sigma=\sigma(x),
$$

independent of $\mathbf{a} \in A$ then

$$
\lim _{\varepsilon \downarrow 0} \hat{u}_{0}^{\varepsilon}=\hat{u}_{0}^{e} \quad \text { uniformly in } x \in \overline{\mathcal{O}}, \quad h=0 .
$$

Proof of (4.11) $h>0$.

Suppose $\varepsilon>0$ is small enough so that

$$
\frac{1}{\varepsilon}>\hat{f}(x, \mathbf{b})+\left[k(\mathbf{a}, \mathbf{b})-k\left(\mathbf{a}, \mathbf{a}^{e}\right)\right]\left(\frac{\alpha}{1-e^{-\alpha h}}\right) \quad \forall x \in \overline{\mathcal{O}}, \quad \mathbf{a} \in A, \quad b \in A-\left\{\mathbf{a}^{e}\right\} .
$$

Using (2.32) from the proof of Theorem 2.1, we have

$$
\begin{aligned}
\hat{u}_{h}^{\varepsilon}(x, \mathbf{a})=E\{ & \left.\int_{0}^{\hat{\theta}_{n} \wedge \hat{\tau}^{n+1}} \hat{f}(\hat{y}(t, \hat{\mathbf{a}}(t)), \hat{\mathbf{a}}(t)) e^{-\alpha t} d t+\sum_{i=1}^{n} k\left(\hat{\mathbf{a}}^{i-1}, \hat{\mathbf{a}}^{i}\right) \mathrm{e}^{-\alpha \hat{\theta}_{i}}\right\} \\
& +E\left\{e^{-\alpha \hat{\theta}_{n}} \hat{u}_{h}^{\varepsilon}\left(\hat{y}\left(\hat{\theta}_{n}, \hat{\mathbf{a}}^{n}\right), \hat{\mathbf{a}}^{n}\right)\right\} .
\end{aligned}
$$


Now for any admissible $\boldsymbol{v}(\cdot)=\mathbf{p}(\cdot) \circ \mathbf{a}(\cdot)$ with $\mathbf{a}(t) \neq a^{e}, t \geqq 0$, we have

$$
0 \leqq \hat{u}_{h}^{e}-\hat{u}_{h}^{\varepsilon} \leqq J_{x \mathbf{a}}(\boldsymbol{v})-\hat{u}_{h}^{\varepsilon}(x, \mathbf{a}) .
$$

By Lemma 4.1 we can consider policies $\hat{\mathbf{a}}(t)$ which switch to $\mathbf{a}^{e}$ in $[\hat{\tau}-h, \hat{\tau}]$ and $\hat{a}(t) \neq \mathbf{a}^{e}, 0 \leqq t<\hat{\tau}-h$. It follows that

$$
0 \leqq \hat{u}_{h}^{e}-\hat{u}_{h}^{e} \leqq \sup _{\mathbf{b} \neq \mathbf{a}^{e}} E\left\{\int_{\hat{\tau}-h}^{\hat{\tau}} \hat{f}(\hat{y}(\mathbf{b}), \mathbf{b}) e^{-\alpha t} d t\right\}
$$

Hence,

$$
0 \leqq \hat{u}_{h}^{e}-\hat{u}_{h}^{\varepsilon} \leqq\left(\sup _{\mathbf{b} \neq \mathbf{a}^{e}}\|\hat{f}(\cdot, \mathbf{b})\|\right)\left(e^{\alpha h}-1\right) / \alpha .
$$

But this can be improved.

Let

$$
n_{h}=\inf \left\{n \geqq 1: \hat{\theta}_{n} \geqq \hat{\tau}-h\right\}, \quad \hat{\theta}_{h}=\hat{\theta}_{n} \quad \text { for } n=n_{h}
$$

Then we can replace $\hat{\tau}-h$ by $\hat{\theta}_{h}$ in (4.17). Since a switching to $\mathbf{a}^{e}$ is assumed to occur in $[\hat{\tau}-h, \hat{\tau}]$ and since (4.6) and (4.14) hold, we have

$$
0 \leqq \sup _{\mathbf{b} \neq \mathbf{a}^{e}}\left(E\left\{\int_{\hat{\theta}_{h}}^{\hat{\tau}} \hat{f}(\hat{y}(\mathbf{b}), \mathbf{b}) e^{-\alpha t} d t-\frac{1}{\varepsilon} \int_{\hat{\theta}_{h}}^{\hat{\tau}} e^{-\alpha t} d t-k\left(\mathbf{b}, \mathbf{a}^{e}\right) e^{-\alpha \hat{\theta}_{h}}\right\}\right) .
$$

This implies

$$
\frac{1}{\varepsilon} E \int_{\hat{\theta}_{h}}^{\hat{\tau}} e^{-\alpha t} d t \leqq \frac{1}{\alpha} \sup _{\mathbf{b} \neq \mathbf{a}^{e}}\|\hat{f}(\cdot, \mathbf{b})\| .
$$

Using this in (4.17) (4.18) with $\hat{\theta}_{h}$ replacing $\hat{\tau}-h$ leads to

$$
0 \leqq \hat{u}_{h}^{e}-\hat{u}_{h}^{\varepsilon} \leqq \frac{1}{\alpha}\left(\sup _{\mathbf{b} \neq \mathbf{a}^{e}}\|\hat{f}(\cdot, \mathbf{b})\|\right)^{2} \varepsilon
$$

Note that (4.22) and (4.18) hold only if $\varepsilon$ and $h$ satisfy (4.14); that is, they are not uniform. In any event (4.22) implies (4.11). QED

Proof of (4.13). $h=0$. Let $\mathbf{a}(t)$ be the optimal policy associated with $\hat{u}_{0}^{\varepsilon}$. It exists by virtue of property (3.7). Write it as

$$
\mathbf{a}(t)=\mathbf{a} \mathbf{1}_{\left[0, \theta_{1}\right)}+\sum_{i=1}^{\infty} \mathbf{a}^{i} \mathbf{1}_{\left[\hat{\theta}_{i}, \hat{\theta}_{i+1}\right)}
$$

where $\hat{\theta}_{i}$ were defined in the proof of Theorem 2.1 (with $h$ set to zero). We define by induction

$$
\tilde{a}^{i}= \begin{cases}\mathbf{a}^{i} & \text { if } \mathbf{a}^{i} \neq \mathbf{a}^{e}, \\ \mathbf{a}^{i-1} & \text { if } \mathbf{a}^{i}=\mathbf{a}^{e},\end{cases}
$$

and if $\mathbf{a} \neq \mathbf{a}^{e}$

$$
\tilde{\mathbf{a}}(t)=\mathbf{a} \mathbf{1}_{\left[0, \hat{\theta}_{1}\right)}+\sum_{i=1}^{\infty} \tilde{\mathbf{a}}^{i} \mathbf{1}_{\left[\hat{\theta}_{i}, \hat{\theta}_{i+1}\right)}
$$


If $\mathbf{a} \neq \mathbf{a}^{\mathbf{e}}$, then

$$
\begin{array}{r}
J_{x \mathbf{a}}(\hat{a}(\cdot))=E_{x}\left\{\int_{0}^{\hat{\theta}_{1} \wedge \hat{\tau}} \hat{f}(\hat{y}(t), \mathbf{a}) e^{-\alpha t} d t+\sum_{i=1}^{\infty} \int_{\hat{\theta}_{i} \wedge \hat{\tau}}^{\hat{\theta}_{i+1} \wedge \hat{\tau}} \hat{f}\left(\hat{y}(t), \hat{a}^{i}\right) e^{-\alpha t} d t\right. \\
\left.+k\left(\mathbf{a}, \hat{\mathbf{a}}^{1}\right) e^{-\alpha\left(\hat{\theta}_{1} \wedge \hat{\tau}\right)}+\sum_{i=1}^{\infty} k\left(\hat{\mathbf{a}}^{i}, \hat{\mathbf{a}}^{i+1}\right) e^{-\alpha\left(\hat{\theta}_{i+1} \wedge \hat{\tau}\right)}\right\}
\end{array}
$$

Since $k(\mathbf{b}, \mathbf{b})=0$ and $k(\mathbf{a}, \mathbf{b})+k(\mathbf{b}, \mathbf{c}) \geqq k(\mathbf{a}, \mathbf{c})$ for each $\mathbf{a}, \mathbf{b}$, and $\mathbf{c}$ in $A$, we have

$$
\begin{array}{r}
J_{x \mathbf{a}}(\hat{a}(\cdot)) \geqq E_{x}\left\{\int_{0}^{\hat{\theta}_{1} \wedge \hat{\tau}} \hat{f}(\hat{y}(t), \mathbf{a}) e^{-\alpha t} d t+\sum_{i=1}^{\infty} \int_{\hat{\theta}_{i} \wedge \hat{\tau}}^{\hat{\theta}_{i+1}} \hat{f}\left(\hat{y}(t), \hat{\mathbf{a}}^{i}\right) e^{-\alpha t} d t\right. \\
\left.+k\left(\mathbf{a}, \tilde{\mathbf{a}}^{1}\right) e^{-\alpha\left(\hat{\theta}_{1} \wedge \hat{\tau}\right)}+\sum_{i=1}^{\infty} k\left(\tilde{\mathbf{a}}^{i}, \tilde{\mathbf{a}}^{i+1}\right) e^{-\alpha\left(\hat{\theta}_{i+1} \wedge \hat{\tau}\right)}\right\} .
\end{array}
$$

Using this and the fact that $\hat{\mathbf{a}}(\cdot)$ is optimal leads to

$$
0 \leqq J_{x \mathbf{a}}(\tilde{\mathbf{a}}(\cdot))-J_{x \mathbf{a}}(\hat{\mathbf{a}}(\cdot)) \leqq \sum_{\mathbf{a}^{i}=\mathbf{a}^{e}} E\left\{\int_{\hat{\theta}_{i} \wedge \hat{\tau}}^{\hat{\theta}_{i+1} \wedge \hat{\tau}}\left[\hat{f}\left(\hat{y}(t), \hat{a}^{i+1}\right)-\hat{f}\left(\hat{y}(t), \mathbf{a}^{e}\right)\right] e^{-\alpha t} d t\right\} .
$$

Since $f \geqq 0$, it follows that for $\mathbf{a} \neq \mathbf{a}^{e}$,

$$
0 \leqq \hat{u}_{0}^{e}(x, \mathbf{a})-\hat{u}_{0}^{\varepsilon}(x, \mathbf{a}) \leqq\left(\sup _{\mathbf{b} \neq \mathbf{a}^{e}}\|\hat{f}(\cdot, \mathbf{b})\|\right)\left(\sum_{\mathbf{a}^{i}=\mathbf{a}^{e}} E\left\{\int_{\hat{\theta}_{i} \wedge \hat{\tau}}^{\hat{\theta}_{i+1^{\wedge}} \hat{\tau}} e^{-\alpha t} d t\right\}\right) .
$$

But using (4.6) gives

$$
\frac{1}{\varepsilon_{\mathbf{a}^{i}=\mathbf{a}}} \sum E\left\{\int_{\hat{\theta}_{\mathbf{i}} \wedge \hat{\tau}}^{\hat{\theta}_{i+1} \wedge \hat{\tau}} e^{-\alpha t} d t\right\} \leqq E\left\{\int_{0}^{\tau} \hat{f}(\hat{y}(t), \hat{\mathbf{a}}(t)) e^{-\alpha t}\right\} \leqq \hat{u}_{0}^{\varepsilon}(x, \mathbf{a}) .
$$

And

$$
0 \leqq \hat{u}_{0}^{\varepsilon}(x, \mathbf{a}) \leqq \min _{\mathbf{b} \in A}\left\{\frac{1}{\alpha}\|\hat{f}(\cdot, \mathbf{b})\|+k(\mathbf{a}, \mathbf{b})\right\} .
$$

It follows that if $\mathbf{a} \neq \mathbf{a}^{e}$, then

$$
0 \leqq \hat{u}_{0}^{e}(x, \mathbf{a})-\hat{u}_{0}^{\varepsilon}(x, \mathbf{a}) \leqq c \varepsilon
$$

with

$$
c=\frac{1}{\alpha}\left(\sup _{\mathbf{b} \neq \mathbf{a}^{e}}\|f(\cdot, \mathbf{b})\|\right)\|\hat{f}(\cdot, \mathbf{a})\| .
$$

This implies (4.13) in case $\mathbf{a} \neq \mathbf{a}^{e}$.

If $\mathbf{a}=\mathbf{a}^{e}$, the argument is much the same. Define

$$
\tilde{\mathbf{a}}(t)=\mathbf{a}^{1} \quad \forall t \in\left[0, \hat{\theta}_{1}\right]
$$

Since (4.30) and (4.31) still hold, we can deduce (4.32) by adding the term

$$
E\left\{k\left(\mathbf{a}^{e}, \mathbf{a}^{1}\right)\left(1-e^{-\alpha\left(\hat{\theta}_{1}^{\wedge} \hat{\tau}\right)}\right)\right\}=\alpha k\left(\mathbf{a}^{e}, \mathbf{a}^{1}\right) E\left\{\int_{0}^{\hat{\theta}_{1^{\wedge}} \hat{\tau}} e^{-\alpha t} d t\right\}
$$

to (4.29). In this case the constant in (4.32) is

$$
c=\alpha\left[\sup _{\mathbf{b} \neq \mathbf{a}^{e}}\|\hat{f}(\cdot, \mathbf{b})\|+\alpha k\left(\mathbf{a}^{e}, \mathbf{b}\right)\right]\left[\min _{\mathbf{b} \boldsymbol{A}} \frac{1}{\alpha}\|\hat{f}(\cdot, \mathbf{b})\|+k\left(\mathbf{a}^{e}, \mathbf{b}\right)\right] .
$$

This completes the argument. QED 
Remark. Using similar techniques, we can consider systems with locally bounded coefficients in an unbounded domain $\mathcal{O}$. All the results can be extended to the associated time-dependent problem.

Acknowledgment. The first named author would like to thank J. S. Baras for useful discussions related to this work.

\section{REFERENCES}

[1] A. Bensoussan AND J. L. Lions, Nouvelle formulation de problèmes de contrôle impulsionnel et applications, C. R. Acad. Sci. Paris, A-276 (1973), pp. 1189-1192.

[2] — Contrôle impulsionnel et systems d'inéquations quasi-variationelles, C. R. Acad. Sci. Paris, A-278 (1974), pp. 747-751.

[3] J. GRuhL, F. SCHWEPPE AND M. RUANE, Unit commitment scheduling of electric power systems, Systems Engineering for Power: Status and Prospects, L. H. Fink and K. Carlsen, eds., US DOE Report NTIS-CONF-750867, 1975, pp. 116-129.

[4] M. RoBIN, Contrôle impulsionnel des processus de Markov, Thèse de Etat, INRIA, Le Chesnay, France, 1977.

[5] J.-L. MENALDI, On the optimal stopping time problem for degenerate diffusions, this Journal, 18 (1980), pp. 697-721.

[6] - On the optimal impulse control problem for degenerate diffusions, this Journal, 18 (1980), pp. 722-739.

[7] A. Bensoussan AND J.-L. LIONS, Inéquations quasi-variationnelles dépendant d'un paramètre, Ann. Scuola Normale di Pisa, ser. IV, (1977), pp. 231-255.

[8] J.-L. MENALDI, A singular perturbation result for variational and quasi-variational inequalities, Nonlinear Anal., 5 (1981), pp. 381-400.

[9] W. E. HOPKINS, JR. AND G. L. BLANKENSHIP, Perturbation analysis of a system of quasi-variational inequalities for optimal stochastic scheduling, IEEE Trans. Automat. Control, AC-26 (1981), pp. 1054-1070.

[10] J.-L. MENAldi, J.-P. QuAdRAT AND E. Rofman, On the role of the impulse fixed cost in stochastic optimal control: An application to the management of energy production, Proc. 10th IFIP Conference on System Modelling Optimization, New York, September 1981, Springer-Verlag, to appear.

[11] A. FRIEDMAN AND P.-L. LIONS, The optimal strategy in the control problem associated with the Hamilton-Jacobi-Bellman equation, this Journal, 18 (1980), pp. 191-198; Corrigendum, 20 (1982), pp. 153-154.

[12] D. W. STROOCK AND S. R. S. VARADHAN, On degenerate elliptic-parabolic operators of second order and their associated diffusions, Comm. Pure Appl. Math., 25 (1972), pp. 651-713. 